\title{
Serum testosterone level after intensity-modulated radiotherapy in low-risk prostate cancer patients: does testicular dose correlate with testosterone level?
}

\author{
Hiromichi Ishiyama • Bin S. Teh • Arnold C. Paulino • \\ Subashini Yogeswaren • Wei-yuan Mai • Bo Xu • \\ E. Brian Butler
}

Received: 4 December 2011 / Accepted: 19 January 2012/Published online: 15 February 2012

(C) Springer-Verlag 2012

\begin{abstract}
Purpose The purpose of this study is to evaluate the relationship between testicular dose and serum testosterone (TE) level in low-risk prostate cancer patients treated with intensity-modulated radiotherapy (IMRT).

Methods and materials This study was conducted as a subset analysis of a previously published prospective clinical trial combining IMRT and gene therapy. To the best of our knowledge, this gene therapy had no significant effect on patient's TE level. Twenty-nine patients with low-risk prostate cancer, who underwent combined treatment of 76 Gy in 35 fractions and gene therapy without any androgen deprivation, were included in this study. No pelvic irradiation was used. TE levels were examined before, during, and after
\end{abstract}

H. Ishiyama • B. S. Teh · A. C. Paulino · B. Xu • E. B. Butler Department of Radiation Oncology,

The Methodist Hospital Cancer Center and Research Institute, Houston, TX, USA

S. Yogeswaren • W.-y. Mai

Department of Radiology/Radiation Oncology,

Baylor College of Medicine,

Houston, TX 77030, USA

H. Ishiyama

Department of Radiology and Radiation Oncology,

Kitasato University School of Medicine,

Sagamihara, Kanagawa, Japan

B. S. Teh $(\triangle)$

Department of Radiation Oncology,

The Methodist Hospital Cancer Center,

6565 Fannin, Ste\#DB1-077,

Houston, TX 77030, USA

e-mail: BTeh@tmhs.org
IMRT. The relationship between mean testicular dose and post-treatment TE levels was assessed.

Results Mean testicular dose was $5.3 \mathrm{~Gy}$ (range, 1.7 to 10.7 Gy). Mean baseline TE level was $310 \mathrm{ng} / \mathrm{dl}$ (range, 113 to $530 \mathrm{ng} / \mathrm{dl}$ ). Before treatment, $66 \%$ of the patients already had hypogonadism. The mean TE levels were significantly decreased at 12, 24, 30, 36 months after treatment when compared to those at baseline $(P<0.05)$. A relatively weak correlation was seen between testicular dose and ratio of post-treatment TE level to baseline. However, this correlation was statistically significant only at 4 months after treatment. There was no significant relationship between TE levels and PSA values.

Conclusion Our study showed decreased post-treatment TE levels of prostate cancer patients after IMRT. A relatively weak correlation was seen between testicular dose and ratio of post-treatment TE level to baseline.

Keywords Testosterone - Radiotherapy - Testicular dose · Testicle $\cdot$ Intensity-modulated radiotherapy

\section{Introduction}

One of the concerns that needs to be resolved among prostate radiotherapy is the incidental dose to the testicles that could lead to impairment of the testosterone (TE) secretion $[1,2]$. This is relevant for techniques that deliver higher scattered testicular doses such as with whole pelvic radiotherapy and with dose-escalated radiotherapy. Intensitymodulated radiotherapy (IMRT) also has the potential of spreading low doses to a large volume of normal tissues. However, there are few studies evaluating integral dose to 
the testicles from IMRT for prostate cancer and whether this has an effect on TE level.

Recently, a controversy occured as to whether iatrogenic hypogonadism confound the results of prostate-specific antigen (PSA) follow-up after radiotherapy [3-5]. King et al. issued their hypothesis as follows: the negative effect on TE production is proportional to the dose to the testicles, and therefore, the time to TE recovery is proportional to testicular dose; and the increase in time to TE recovery leads to a delay in biochemical failure [4]. However, their hypothesis is not proven at this time.

Aside from the abovementioned controversy, some consequences of hypogonadism also caused by impairment of TE secretion would be significant including anemia [6], osteoporosis [7], increased risk of cardiac events [8], declining mental acuity, muscle atrophy, lack of libido, erectile dysfunction, obesity, depression, hypertension, diabetes, and degradation in quality of life [9].

Thus, exploring the relationship between testicular dose and hypogonadism after prostate radiotherapy is a crucial issue not only for clinical consequences but also for controversy of confounding bias of PSA follow-up. The purpose of this study was to evaluate the relationship between the testicular dose and post-treatment TE level in low-risk prostate cancer patients treated with combined IMRT and gene therapy.

\section{Methods and materials}

This study was conducted as a subset analysis of a previously published phase I/II trial of combined IMRT and herpes simplex virus thymidine kinase gene therapy [10, 11]. From data of 33 patients with low-risk prostate cancer (T1-T2a, Gleason score $<7$, pre-treatment PSA $<10$ ) treated

Table 1 Patient characteristics $(n=29)$

\begin{tabular}{lc}
\hline Age (years) & $64(51-78)$ \\
Initial PSA (ng/ml) & $4.7(2.0-9.4)$ \\
Gleason score & 2 \\
5 & 27 \\
6 & \\
T stage & 16 \\
T1c & 13 \\
T2a & $64.7(38.6-112.9)$ \\
Prostate volume $\left(\mathrm{cm}^{3}\right)$ & \\
Testicular volume $\left(\mathrm{cm}^{3}\right)$ & $35.9(10.0-141.1)$ \\
Right & $46.7(19.7-112.2)$ \\
Left & $310(113-530)$ \\
Pre-treatment serum testosterone level (ng/dl) & \\
\hline
\end{tabular}

Values are number or median (range) without any androgen deprivation, 29 patients were included in this study. The other four patients were excluded because of lack of sufficient data. Patients' characteristics are shown in Table 1.

A mean dose of 76 Gy (prescribed dose of $70 \mathrm{~Gy}$ in 2-Gy fractions) in the form of IMRT was delivered to the prostate in 35 fractions using the NOMOS Peacock system (NOMOS, Cranberry Township, PA). FifteenMV photons were used. None of the patients received pelvic lymphatic irradiation. Radiotherapy was initiated $48 \mathrm{~h}$ after the first gene vector injection. The techniques have been described in detail previously $[12,13]$. In brief, the patients were treated prone in a customized vacuum bag fitted into a treatment box for the purpose of immobilization. A rectal balloon was placed each day during treatment to minimize prostate motion and to decrease rectal volume receiving high-dose radiation [12-14]. In addition to prostate and normal tissues including the rectum, bladder, and femoral heads, both left and right testicles were outlined for every patient. Not only testicles but also epididymis, distal parts of the spermatic cord, and some part of the fascia were inevitably contoured as testicles because these organs are difficult to be distinguished in $\mathrm{CT}$ images. The mean doses for right, left, and total testicular volumes were calculated.

Serum TE and PSA levels were evaluated before initiation of treatment, during the sixth week of IMRT, as well as at 1.5, $4,8,12,18,24,30,36$ months post-IMRT. All TE measurements were performed in one laboratory, using radioimmunoassay with a normal range of 350 to $720 \mathrm{ng} / \mathrm{dl}$. PSA measurements were also done at the same laboratory.

Statistical analysis was performed using paired $t$ test for comparing post-treatment TE levels on each evaluation with the respective baselines. Pearson correlation coefficient was calculated for evaluating correlations between serum TE and related factors. $P$ values less than 0.05 were considered statistically significant.

\section{Results}

Treatment result

Serum PSA levels fell with time after treatment when compared to the baseline PSA (data not shown). One patient had PSA failure (nadir +2.0) on 24 months after treatment. The other patients had no biochemical or clinical recurrence during the follow-up duration. There was no significant relationship between post-treatment TE levels and PSA levels. Mean testicular dose was $5.3 \mathrm{~Gy}$ (range, 1.7 to 10.7 Gy). There was no significant difference between the dose of the right testis and that of the left testis. 


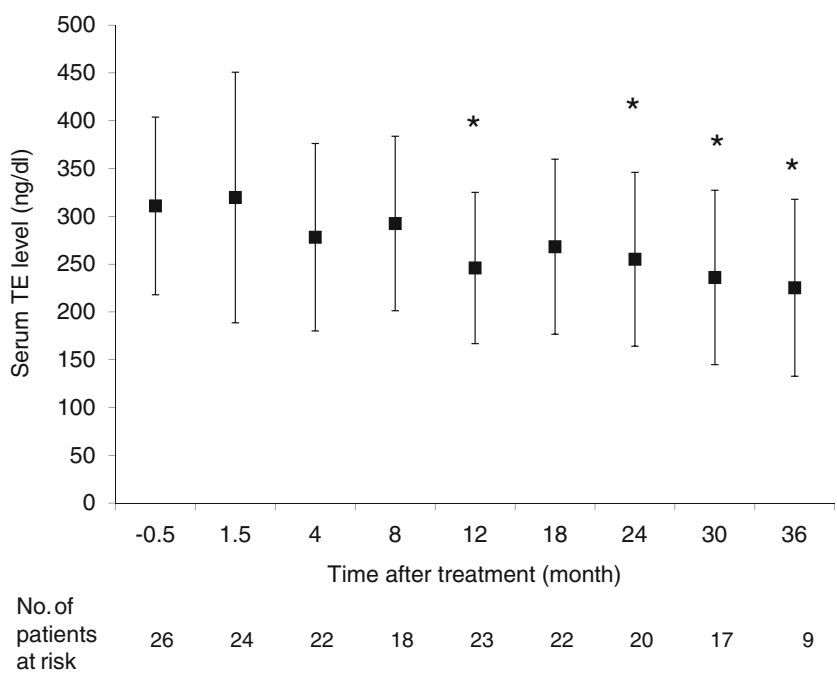

Fig. 1 Change of serum testosterone level before and after treatment. Testosterone levels tended to decline over time. At 12, 24, 30, and 36 months after treatment, the testosterone level significantly declined from baseline level (asterisk). Squares represent mean. Error bars represents standard deviation. TE testosterone

\section{Post-treatment TE level}

The mean TE levels for the sixth week during treatment, 1.5, 4, $8,12,18,24$, and 36-months after treatment were 312, 326, 285, 292, 240, 264, 244, and $225 \mathrm{ng} / \mathrm{dl}$ respectively (Fig. 1). Post-treatment TE levels tended to decline over time, and

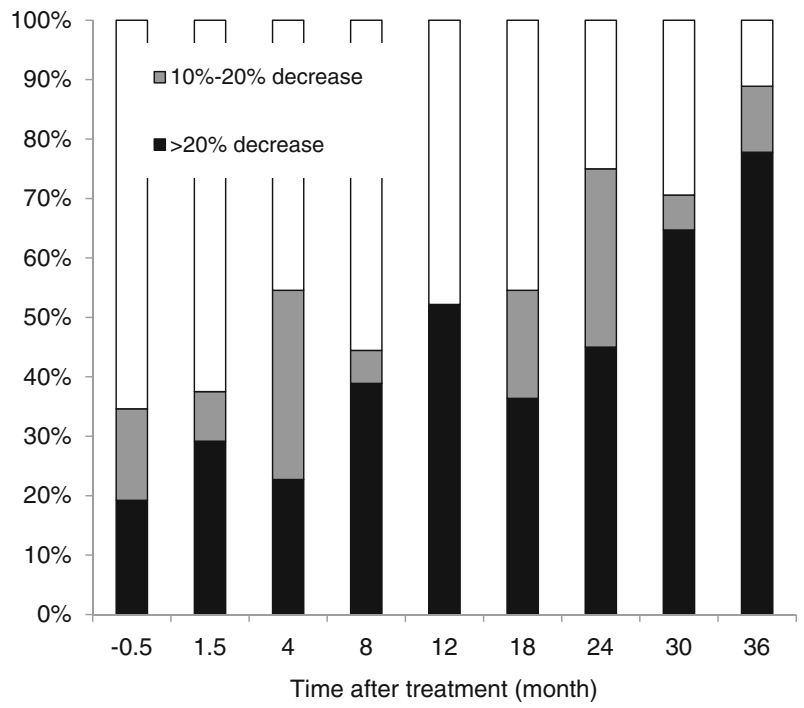

No. of

$\begin{array}{llllllllll}\text { patients } & 26 & 24 & 22 & 18 & 23 & 22 & 20 & 17 & 9\end{array}$ at risk

Fig. 2 Percentages of patients with reduced serum testosterone level. During radiotherapy, $30 \%$ of the patients already had over $10 \%$ reduction of serum testosterone levels. Proportion of patient with reduced testosterone levels increased over time

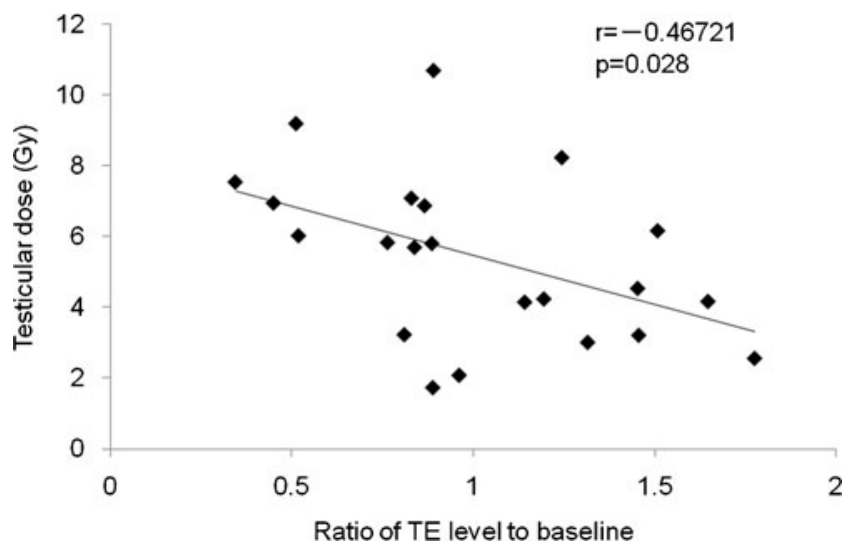

Fig. 3 Relationship between testicular dose and ratio of post-treatment testosterone level to baseline. Mean testicular doses negatively correlated to ratio of post-treatment testosterone level to baseline at 4 months after treatment. $T E$ testosterone

significantly declined compared to baseline at $12,24,30$, and 36 months after treatment $(P<0.05)$. Figure 2 shows percentages of over $20 \%$ or $10-20 \%$ reduction of post-treatment TE level from baseline. Many patients showed significant decreases of TE level over time. Even at baseline, already $66 \%$ of the patients had hypogonadism, and $66 \%, 71 \%$, $71 \%, 72 \%, 90 \%, 82 \%, 80 \%, 88 \%$, and $78 \%$ of the patients were at the hypogonadal level on the sixth week during the treatment, 1.5, 4, 8, 12, 18, 24, and 36 months after treatment.

\section{Ratio of post-treatment TE levels to baseline}

In addition to actual TE levels, ratio of post-treatment TE levels to baseline was assessed for statistical analysis because the baseline TE level had a wide range (Table 1). When comparing TE level at 4 months after treatment with that before treatment, a statistically significant correlation between testicular dose and ratio of post-treatment TE level to baseline was seen $(r=-0.467, P=0.03$; Fig. 3 ). The same trends were shown except for 36 months that had only nine patients for analysis (Table 2).

Table 2 Correlation coefficients between testicular dose and ratio of post-treatment TE level to baseline

$r$ correlation coefficient ${ }^{\text {a }}$ Statistically significant

\begin{tabular}{lll}
\hline & $r$ & $P$ value \\
\hline 6th week & -0.158 & 0.44 \\
1.5 months & -0.178 & 0.40 \\
4 months & -0.467 & $0.03^{\mathrm{a}}$ \\
8 months & -0.131 & 0.60 \\
12 months & -0.128 & 0.56 \\
18 months & -0.223 & 0.32 \\
24 months & -0.384 & 0.09 \\
30 months & -0.124 & 0.64 \\
36 months & 0.251 & 0.51 \\
\hline
\end{tabular}




\section{Discussion}

Our study showed decreased post-treatment TE levels of prostate cancer patients after IMRT. A relatively weak correlation was seen between testicular dose and ratio of posttreatment TE level to baseline.

King et al. [4] have proposed the hypothesis that the negative effect on TE production is proportional to the dose to the testicles, and testicular dose is associated with lower TE levels and delays in time to recover normal TE levels. As a result, diagnosis of PSA recurrence might be confounded by change of TE level. In this hypothesis, a proportional relationship between testicular dose and TE level is crucial. Our study showed a weak relationship between testicular dose and TE level, and some part of hypothesis of King et al. was supported by our study. However, recovery time of TE levels could not be assessed with limited follow-up duration of our study. In addition, there is no evidence for relationship between recovery of TE level and PSA recurrence at this time [15]. Thus, there is no sufficient evidence for drawing a conclusion about the controversy whether iatrogenic hypogonadism confounds the results of PSA follow-up after radiotherapy at this time.

In the general population, the incidence of hypogonadism is $20 \%$ in men over $60,30 \%$ in men over 70 , and $50 \%$ in men over 80 years of age [16]. However, $66 \%$ of our patients with median age of 64 years had hypogonadism before treatment. It was three times as many as the normal population. This fact suggested that the baseline TE level of our patients was affected by factors other than their age, and also suggested that the relationship between testicular dose and TE level may be confounded by factors other than the analyzed ones in this study. It is reported that TE level can be affected by various factors such as body mass index, smoking, coffee consumption, having a stroke, waist circumference, physical activity, depression [17], time of blood collection, sex hormone-binding globulin concentration, diabetes mellitus, hyper- and hypothyroidism [18], having a cancer diagnosis [19], annual number of outpatient visits, and annual outpatient costs [20]. In this subset analysis, however, it was difficult to avoid these confounding factors because of limitation of available data. In addition, our sample size was relatively small. Follow-up period was not sufficiently long. Further study is recommended with sufficient consideration of correlated factors to TE level.

Conflicts of interest No actual or potential conflicts of interest exist in this report.

\section{References}

1. Rowley MJ, Leach DR, Warner GA et al (1974) Effect of graded doses of ionizing radiation on the human testis. Radiat Res 59:665-678

2. Tomic R, Bergman B, Damber JE et al (1983) Effects of external radiation therapy for cancer of the prostate on the serum concentrations of testosterone, follicle-stimulating hormone, luteinizing hormone and prolactin. J Urol 130:287-289

3. Roach M 3rd, Lawton CA, Chen J (2010) To treat pelvic nodes or not? Greater testicular scatter does not explain the results of randomized trials. J Clin Oncol 28:e450-e452

4. King CR, Kapp DS (2009) To treat pelvic nodes or not: could the greater testicular scatter dose from whole pelvic fields confound results of prostate cancer trials? J Clin Oncol 27:6076-6078

5. King CR, Maxim PG, Hsu A et al (2010) Incidental testicular irradiation from prostate IMRT: it all adds up. Int J Radiat Oncol Biol Phys 77:484-489

6. Strum SB, McDermed JE, Scholz MC et al (1997) Anaemia associated with androgen deprivation in patients with prostate cancer receiving combined hormone blockade. $\mathrm{Br} \mathrm{J}$ Urol 79:933-941

7. Shahinian VB, Kuo YF, Freeman JL et al (2005) Risk of fracture after androgen deprivation for prostate cancer. N Engl J Med 352:154-164

8. D'Amico AV, Denham JW, Crook J et al (2007) Influence of androgen suppression therapy for prostate cancer on the frequency and timing of fatal myocardial infarctions. J Clin Oncol 25:2420-2425

9. Bruchovsky N, Klotz L, Crook J et al (2008) Quality of life, morbidity, and mortality results of a prospective phase II study of intermittent androgen suppression for men with evidence of prostate-specific antigen relapse after radiation therapy for locally advanced prostate cancer. Clin Genitourin Cancer 6:46-52

10. Teh BS, Ayala G, Aguilar L et al (2004) Phase I-II trial evaluating combined intensity-modulated radiotherapy and in situ gene therapy with or without hormonal therapy in treatment of prostate cancer-interim report on PSA response and biopsy data. Int $\mathrm{J}$ Radiat Oncol Biol Phys 58:1520-1529

11. Teh BS, Aguilar-Cordova E, Kernen K et al (2001) Phase I/II trial evaluating combined radiotherapy and in situ gene therapy with or without hormonal therapy in the treatment of prostate cancers - a preliminary report. Int $\mathrm{J}$ Radiat Oncol Biol Phys 51:605-613

12. Teh BS, Woo SY, Mai WY et al (2002) Clinical experience with intensity-modulated radiation therapy (IMRT) for prostate cancer with the use of rectal balloon for prostate immobilization. Med Dosim 27:105-113

13. Teh BS, Mai WY, Uhl BM et al (2001) Intensity-modulated radiation therapy (IMRT) for prostate cancer with the use of a rectal balloon for prostate immobilization: acute toxicity and dosevolume analysis. Int J Radiat Oncol Biol Phys 49:705-712

14. Smeenk RJ, Teh BS, Butler EB et al (2010) Is there a role for endorectal balloons in prostate radiotherapy? A systematic review. Radiother Oncol 95(3):277-282

15. Roach M 3rd, Bae K, Lawton C et al (2010) Baseline serum testosterone in men treated with androgen deprivation therapy and radiotherapy for localized prostate cancer. Int J Radiat Oncol Biol Phys 78(5):1314-1322

16. Harman SM, Metter EJ, Tobin JD et al (2001) Longitudinal effects of aging on serum total and free testosterone levels in healthy men. Baltimore Longitudinal Study of Aging. J Clin Endocrinol Metab $86: 724-731$ 
17. Morsink LF, Vogelzangs N, Nicklas BJ et al (2007) Associations between sex steroid hormone levels and depressive symptoms in elderly men and women: results from the Health ABC study. Psychoneuroendocrinology 32:874-883

18. Bhasin S, Cunningham GR, Hayes FJ et al (2010) Testosterone therapy in men with androgen deficiency syndromes: an Endocrine Society clinical practice guideline. J Clin Endocrinol Metab 95:2536-2559
19. Svartberg J, Midtby M, Bonaa KH et al (2003) The associations of age, lifestyle factors and chronic disease with testosterone in men: the Tromso Study. Eur J Endocrinol 149:145-152

20. Haring R, Baumeister SE, Volzke H et al (2010) Prospective association of low serum total testosterone levels with health care utilization and costs in a population-based cohort of men. Int $\mathrm{J}$ Androl 33(6):800-809 